\title{
Zuchtversuche mit japanischen Tanzmäusen und europäischen Laufmäusen.
}

\author{
Von \\ Privatdozent Dr. Viktor Hammerschlag. \\ (Aus der Biologischen Versuchsanstalt in Wien.)
}

Mit 3 Fignren im Text.

Eingegangen am 13. September 1911.

Im Nachstehenden berichte ich über meine Zuchtversuche mit japanischen Tanzmäusen und weißen europäischen Lanfmäusen.

Bevor ich aber die Versuche selbst und ibre Resultate anführe, sei kurz der Zweck dieser Versuche und der Weg skizziert, auf welchem ich zu diesen Zuchtversuchen gelangt bin.

Ich beschäftige mich seit Jahren mit der Ätiologie, der Feststellung des klinischen Bildes und der verschiedenen Begleiterscheinungen der von mir sogenannten hereditär-degenerativen Taubstummheit des Menschen.

Es war mir nun darum zu tun, die Gesetze, nach denen sich diese Form der Taubstummheit vererbt, festzustellen. Nun ist es eine bekannte Tatsache, daß wir ein Analogon dieser Taubstummheit bei verschiedenen domestizierten Tieren antreffen, und zwar sowohl bei Hunden, Katzen und Mäusen. Die Mäuse, als diejenigen Tiere, welche sich am raschesten vermehren und daher in verbältnismäBig kurzen Zeiträumen eine genügend große Nachzucht geben, waren selbstverständlich für diese Versuche das geeignetste Objekt.

Ich begann mit meinen Versuchen im Sommer des Jahres 1906. Vor diesem Zeitraum batte ich allerdings bereits einzelne Versuche vorgenommen, die aber von mir obne Kenntnis der Mendelschen Vererbung:gesetze unternommen worden waren. Erst im Sommer 1906 nnternahm ich systematische, auf eine Nachprïfung der Mendelschen Vererbungsgesetze hinzielende Versuche. 
Die Stammtiere waren eine männliche japanische (d. i. eine schwarz-weiß gefleckte) Tanzmaus und zwei weibliche albinotische Laufmäuse. Von dieser Stammgeneration stammte eine Anzahl grauer einfärbiger Laufmäuse. Davon kamen acht Individuen in das geschlechtsreife Alter. Diese acht Mäuse wurden nun zu einer Zucht vereinigt und die von dieser Generation stammenden Jungen wurden stets solange bei den Eltern belassen, bis sie sich selbstständig ernähren konnten, sodann herausgenommen und nach dem gleich zu beschreibenden Schema in verschiedenen Zuchten verteilt.

Wie wir aus der Literatur wissen, sind die Farbe bzw. die Farblosigkeit, ferner die Scheckung bzw. die Einfärbigkeit und der Tanzmauscharakter bzw. der Laufmauscharakter Eigenschaften, die sich unabhängig voneinander vererben.

In unserm Falle hatten wir es nun mit einer schwarz-weiß gescheckten und einer vollkommen albinotischen Maus zu tun. Die schwarz-weiß gescheckte Maus repräsentierte den Charakter der Scheckung sowie den Charakter des Farbbesitzes. Die weiße Maus repräsentierte den Charakter der Farblosigkeit (Albinismus) sowie den Charakter der Einfärbigkeit. (Zwar kann eine albinotische Maus latent den Charakter der Scheckung an sich tragen, und wenn man derartige weiße Mäuse zur Aufzucht verwenden würde, käme man in Gefahr, falsche Resultate zu erhalten. In unserm Falle war es allerdings sicher, daß die albinotischen Mäuse nicht von gescheckten Mäusen abstammten.)

Bei der Kreazung zweier, wie oben beschriebener Tiere, die in drei Merkmalen konstant differieren, müssen nun bei den Kreuzungsprodukten eine ganz bestimmte Anzahl verschiedener Merkmalskombinationen wieder auftreten, und zwar müssen wir erhalten:

1) gefärbte, einfärbige Laufmäuse und ebensolche Tanzmäuse;

2) albinotische Laufmäuse und albinotische Tanzmänse;

3) gescheckte Laufmäuse und gescheckte Tanzmäuse.

Weiters müssen sich die einfärbigen sowie die gescheckten Laufbzw. Tanzmäuse wieder spalten in graue und schwarze einfärbige Lauf- bzw. Tanzmäuse, sowie in grau-weiß und schwarz-weiß gescheckte Lauf- bzw. Tanzmäuse. Wir haben sonach zehn verschiedene Arten zu erwarten und diese zehn Arten wurden bis auf eine einzige Art in der zu beschreibenden Zuchtreihe auch wirklich erzielt.

Die acht grauen Bastarde gaben insgesamt 117 Junge. Dazu 
kamen sechs weitere Junge, die wenige Tage nach der Geburt eingingen, so daß ihr Tanz- bzw. Laufmauscharakter nicht hatte festgestellt werden können. Drei von ihnen waren grau, drei weiß. Diese 117 (123) Individuen der ersten Generation zerfielen nun in folgende zehn Unterabteilungen:

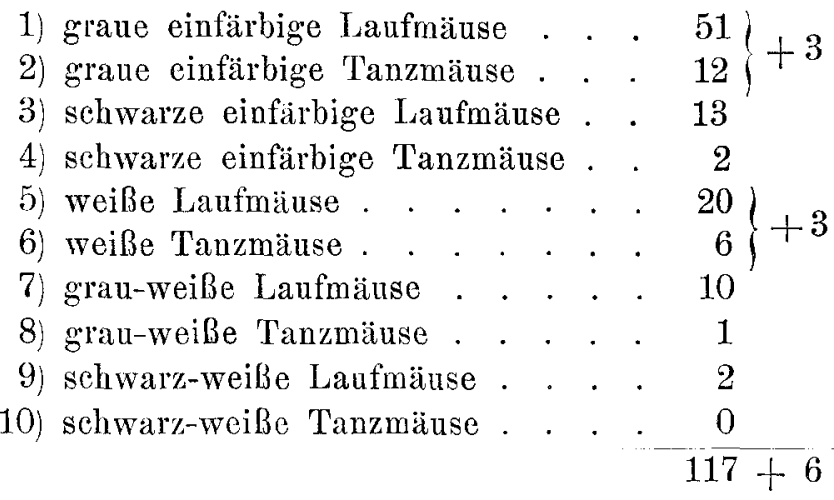

In Parenthese sei hier bemerkt, daß die schwarz-weißen Tanzmäuse, die ich bei dieser Zuchtreihe nicht erzielte, bei einer andern Zuchtreihe anftraten. Es waren sehr debile Individuen, die nicht ins geschlechtsreife Alter kamen.

Auf den Ausgangspunkt zurüekgreifend, sei hier kurz bemerkt, daß der Habitus der Bastardgeneration uns erkennen läßt, welche Merkmale sich dominierend und welche sich recessiv verhalten werden. Der Bastard ist bei den von mir gewählten Rassen stets eine einfürbige grane Lanfmaus. Es ist demnach zu erwarten, daß das Merkmal Einfärbigkeit und das Merkmal Laufmauscharakter die dominierenden Merkmale sind. Das verbält sich nun, wie wir bereits aus der Literatur wissen und wie auch ich wieder konstatieren konnte, tatsächlich so. Daß dem so ist, geht aus den weiteren Resultaten meiner Zuchtversuche hervor. Es wurden nämlich die Invividuen der ersten Generation nach den eben skizzierten Merkmalen in zehn gesonderte Zuchten verteilt. Das Schicksal dieser Zuchten war verschieden. Manche von ihnen gingen zu rasch ein und ergaben kein genügendes Material. Andre wieder ließen sich durch eine genügend lange Zeit mit gutem Erfolge fortzüchten. In Kürze ließe sich das Resultat folgendermaßen feststellen:

Albinotische Tiere ergaben miteinander fortgeziichtet immer nur albinotische Nachzucht. 
है
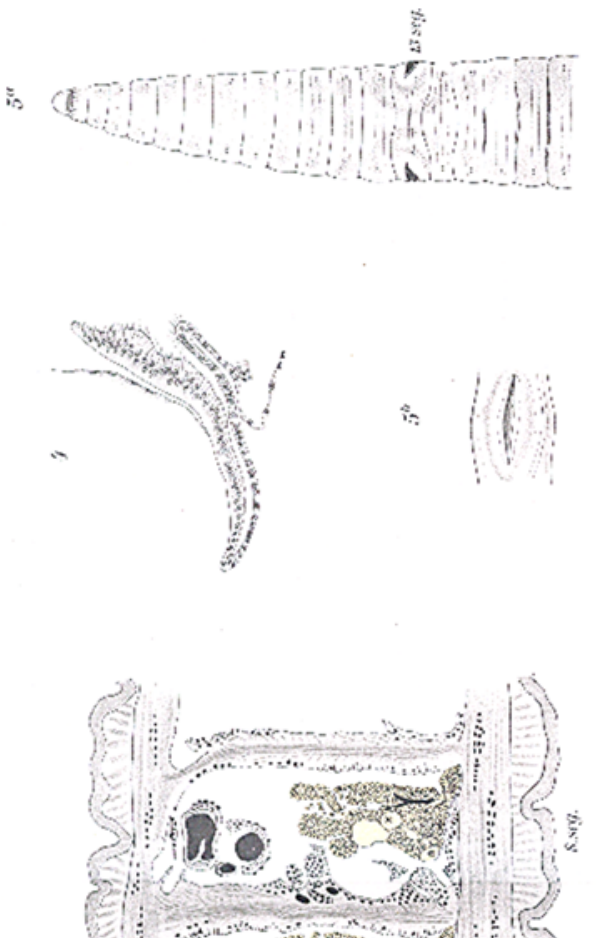

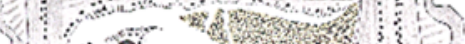

3100 mon

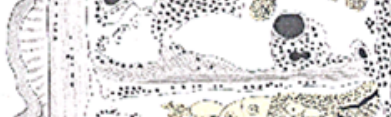

2. 0.10
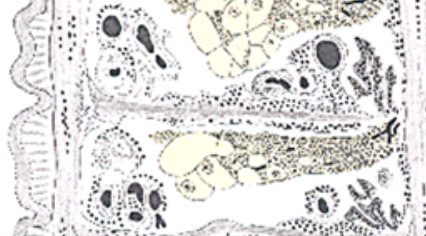

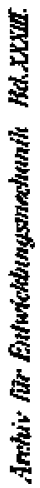

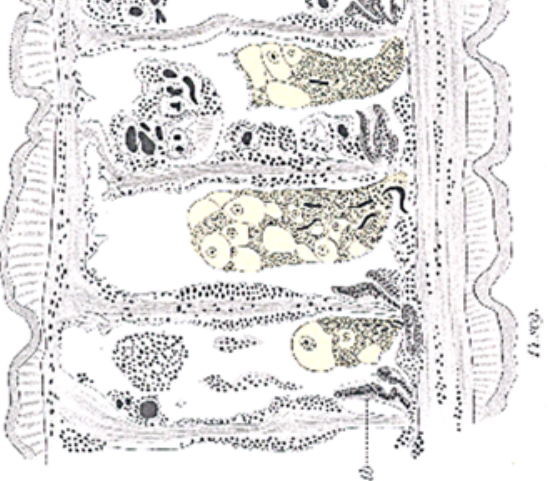

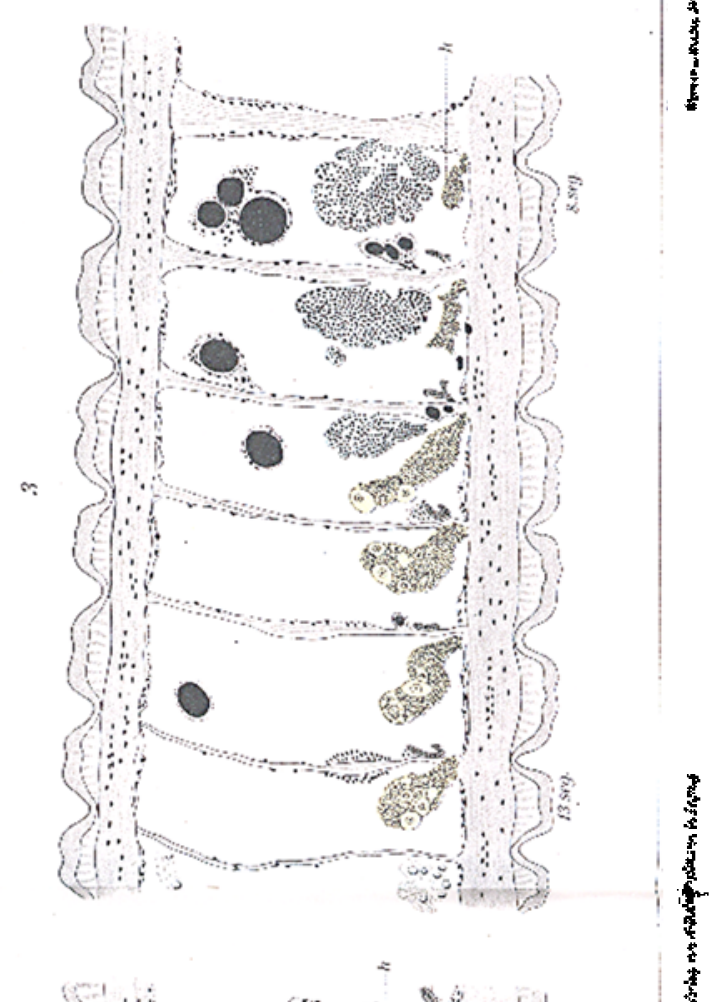

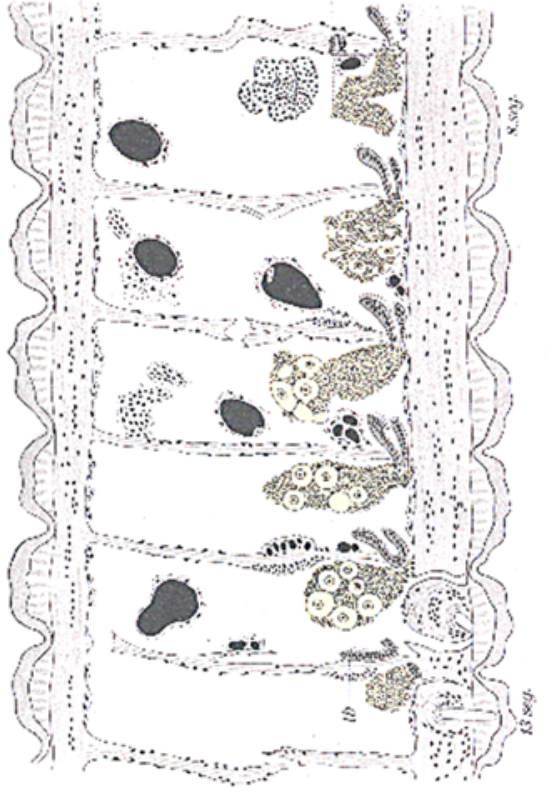


Tanzmäuse ergaben mit Tanzmäusen gekreuzt immer nur Tanzmäuse, und gescheckte Tiere untereinander fortgezüchtet ergaben stets nur Schecken. Ferner erwies sich die graue Farbe der schwarzen übergeordnet, insofern, als man aus grauen Tieren stets auch schwarze züchten konnte. Nie aber erhielt ich von schwarzen Tieren graue Nachkommen. Alle diese Resultate wurden von meinen Vorgängern bereits erzielt.

Während nun in diesem Punkte das Mendelsehe Gesetz seine volle Bestätigung fand, traten in bezug auf das Zahlenverhältnis der dominierenden bzw. recessiven Merkmale Abweichungen vom Mendelschen Gesetze auf. Betrachten wir zunächst das Verhältnis der Läufer zu den Tänzern. Dasselbe stellte sich wie $96 \mathrm{zu} \mathrm{21,} \mathrm{d.} \mathrm{i.}$ wie $4,57: 1$. Wir sehen hier ein bedeutendes Überwiegen der Laufmäuse. Diese Abweichung von der Mendelschen Formel (3:1) wurde iibrigens von allen Experimentatoren konstatiert, ohne daß bisher eine ausreichende Erklärung für diesen Umstand gefunden wurde.

Das Verhältnis der gefärbten Mäuse zu den albinotischen stellte sich wie $94: 29$, d. i. wie $3,24: 1$. Hier zejgt sich demnach eine ziemlich weitgehende Übereinstimmung mit dem theoretisch zu erwartenden Resultat.

Einen ganz besonderen Mangel an Übereinstimmung zeigt aber das Verhältnis der einfärbigen Tiere zu den gescheckten. Wenn wir die beiden Merkmalspaare Scheckung und Einfärbigkeit sowie Farbe und Farblosigkeit kombinieren, so stellt sich theoretisch das Zahlenverhältnis wie $9: 3: 4$, das heißt, auf neun einfärbige Tiere kommen drei gescheckte und vier Albinos. Bei uns stellte sich das Verhältnis wie $81: 13: 29$, das ist wie $9: 1,44: 3,22$.

Wir sehen sonach wohl eine Übereinstimmung in bezug auf die Progression, indem die Einfärbigen in der Mehrzahl, die Schecken in der Mindestzahl auftreten und die Albinos zwischen beiden die Mitte halten. Es zeigt sich aber auch hier ein sebr bedeutender Ausfall an Schecken und, wie schon erwähnt, ein kleiner Ausfall an Albinos.

Anhangsweise sei hier kurz ein Vorkommnis erwähnt, welches im Laufe der Zuchtversuche beobachtet wurde. Es bezieht sich auf die Vererbbarkeit von Traumen.

Einem Tanzmausmännchen, welches einer größeren Zucht von gewöhnlichen japanischen Tanzmäusen angehörte, ging durch Ab- 
Zuchtversuche mit japanischen Tanzmäusen und europäischen Laufmäusen. 343 schnürung ein Hinterbein verloren. Das Bein wurde gangränös und starb ab. Von diesem Männchen stammte ein Warf von vier gleich-

Fig. 1.

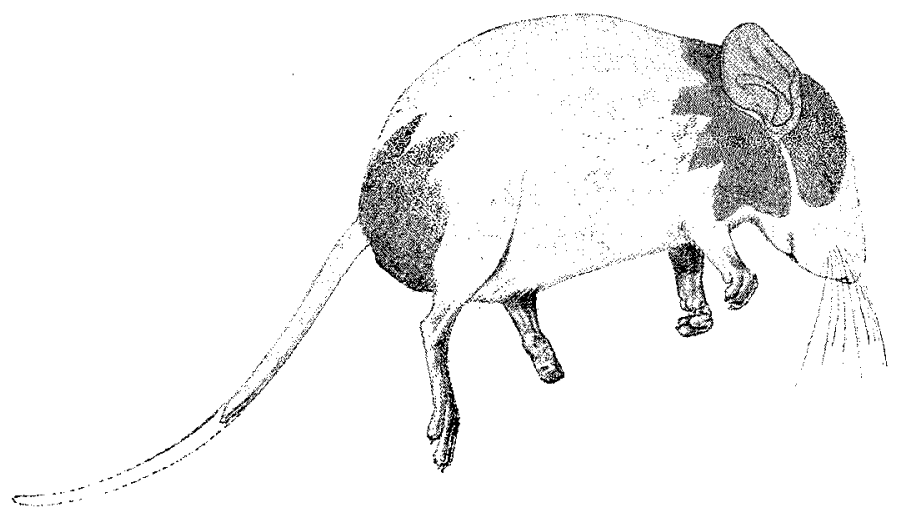

Fig. 2.

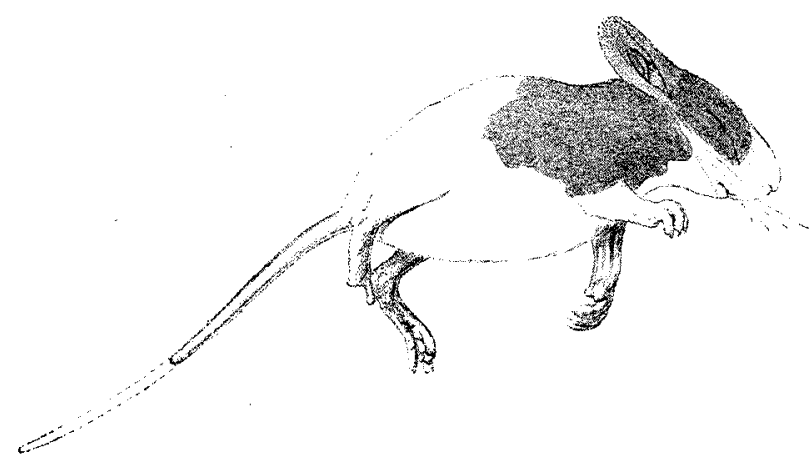

Fig. 3.

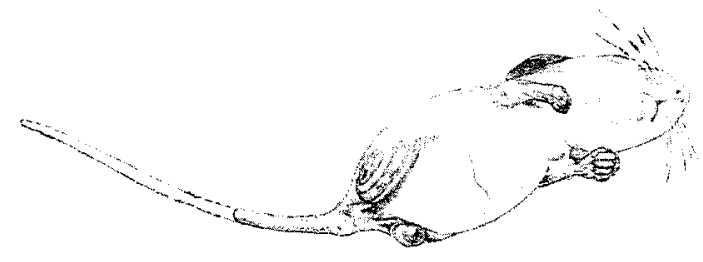

falls japanischen Tanzmüusen, die alle mißgebildet waren. Zwei von diesen mißgebildeten Jungen erreichten nicht das geschlechtsreife Alter, zwei konnte ich aufziehen. 
344 Viktor Hammerschlag, Zuchtversuche mit japanischen Tanzmäusen usw.

(Die Abbildungen auf S. 343 zeigen die an dreien dieser Tiere zu beobachtenden Mißbildungen.)

Sie laufen im wesentlichen auf eine Verkürzung des Schwanzes, sowie auf eine Mißbildung verschiedener Extremitäten hinaus. Ein ähnliches Resultat konnte später nie mehr erzielt werden. Sowohl die beiden ins geschlechtsreife Alter gelangten mißgebildeten Mäuse, die zufälligerweise ein Pärchen bildeten, als auch andre Mäuse, an denen ich mittels Seidenfaden einzelne Extremitäten abschnürte, wurden zur Nachzucht verwendet, ohne daß jemals bei den Produkten congenitale Mißbildungen aufgetreten wären. 